\title{
Alternative pathway complement activation in secondary thrombotic microangiopathies: is genetics the smoking gun?
}

\author{
Joaquim Calado \\ Nephrology Department, Centro Hospitalar e Universitário de Lisboa Central. \\ ToxOmics, Nova Medical School.
}

Two different reports in the current PJHN highlight the mist that surrounds the role of the alternative pathway complement activation in secondary forms of thrombotic microangiopathies (TMA). Depending on whether such a role is validated or not, what is at stake is a more disseminated use of anti $\mathrm{C} 5$ therapy. The latter has been restricted to the atypical forms of Hemolytic Uremic Syndrome (aHUS), which are the TMAs caused by dysregulation of the alternative complement pathway ${ }^{1}$.

According to accepted guidelines, aHUS diagnosis is to be considered only after secondary forms of TMA have been excluded ${ }^{1}$. The challenge presented by the well-documented cases reported by Teixeira AC, et $a l^{2}$ and Diniz $\mathrm{H}$, et $a^{\beta}$ is that even in immune-mediated glomerulonephritis, defects of the alternative pathway complement activation may coexist together with the activation of the classical (by IgG or IgM containing immune complexes) or lectin (by galactose deficient IgA1 containing immune complexes) complement pathways ${ }^{4}$.

In both cases, the hematological evidence of an ongoing microangiopathic Coombs-negative hemolytic anemia is undisputable. In addition, the histopathological findings of TMA are detailed, affecting arterioles in the case of IgA nephropathy ${ }^{3}$, while glomerular capillaries are reported to be involved in the hepatitis $\mathrm{C}$ virus-associated membranoproliferative glomerulonephritis (MPGN) description². However, for the latter (cryoglobulinemic), one must question if what is displayed are not indeed the hyaline thrombi seen in immunocomplex-mediated MPGN rather than the platelet and fibrinogen containing capillary thrombus in TMA. Ultrastructural evaluation of the renal biopsy is warranted before a definite conclusion can be drawn here.

Regarding the phenotype studies on complement pathways, $C^{\prime} 3$ levels are not detailed in the IgA nephropathy case, although are assumed to be normal. This is relevant, not only because $C^{\prime} 3$ consumption is associated with a worst prognosis in IgA nephropathy ${ }^{5}$ but also because C' 3 levels could help in establishing geno-phenotype correlations in face of the $\mathrm{CFH}$ and $\mathrm{C} 3$ gene alleles identified in this individual. In the cryoglobulinemic report, and even if the decreased levels for $C^{\prime} 3$ and $C^{\prime} 4$ are in line with the detected cryoglobulinemia and classical pathway activation, the absence of anti-FH antibodies determination hampers the pathogenic validation of the CFHR3-1 locus homozygous deletions, as acknowledged by the authors in the first place.

In both cases, as expected (or unexpectedly), genetic testing was performed in the search for pathogenic variants in the genes coding for proteins involved in the alternative complement pathway ${ }^{6}$. And the results could not have been more contrasting. While in the IgA case, the CFH allele (c.3611G>A, p.Gly1204Glu) in heterozygocity is one of unquestionable pathogenicity and can, alone, account for a TMA/aHUS phenotype, in the cryoglobulinemic case, allele pathogenicity validation is questionable, as acknowledged by the authors in the first place. It is disputable if missense variants in the complement factor H-related proteins (encoded by the CFHR genes) are of any significance in aHUS, while the pathogenicity of the CFHR3-1 homozygous deletion, a polymorphic allele with a frequency in excess of $15 \%$ in western populations ${ }^{7}$, cannot be inferred in the absence of documented anti-FH antibodies.

In light of these illustrative cases, the question remains if we should test all TMAs for pathogenic variants in the genes of interest? My personal view is that we should. If found, the impact of these is of the utmost importance in, for instance, transplantation options and outcomes. But this is valid as long as clinicians are able to critically appraise human molecular genetic findings.

Disclosure of potential conflicts of interest: none declared

\section{References}

1. Azevedo A, Faria B, Teixeira $\mathrm{C}$ et al. Portuguese consensus document statement in diagnostic and management of atypical hemolytic uremic syndrome. Port J Nephrol Hypert 2018; 32(3): 211-232.

2. Teixeira AC, Pinto $\mathrm{H}$, Afonso $\mathrm{N}$ et al. Cryoglobulinemic HCV-related membranoproliferative glomerulonephritis with TMA - secondary HUS or infection-triggered aHUS? Port J Nephrol Hypert 2019; 33(4): 223-226

3. Diniz H, Bandeira M, Nunes AT et al. IgA Nephropathy with thrombotic microangiopathy: is this secondary thrombotic microangiopathy or IgA nephropathy-triggered atypical Hemolytic Uremic Syndrome? Port J Nephrol Hypert 2019; 33(4): 207-211.

4. Maillard N, Wyatt RJ, Julian BA et al. Current understanding of the role of complement in IgA nephropathy. J Am Soc Nephrol. 2015; 26(7):1503-12.

5. Kim SJ, Koo HM, Lim BJ et al. Decreased circulating C3 levels and mesangial C3 deposition predict renal outcome in patients with IgA nephropathy. PLoS One. 2012; 7(7):e40495.

6. Fidalgo T, Martinho P, Pinto CS et al. Combined study of ADAMTS13 and complement genes in the diagnosis of thrombotic microangiopathies using next-generation sequencing. Res Pract Thromb Haemost. 2017;1:69-80.

7. Holmes LV, Strain L, Staniforth SJ et al. Determining the population frequency of the CFHR3/CFHR1 deletion at 1q32. PLoS ONE 8(4): e60352.

\section{Correspondence to:}

Joaquim Calado, MD, PhD

Nephrology Department, Centro Hospitalar de Lisboa Central

E-mail: jcalado@nms.unl.pt 